\title{
Lemierre's Syndrome with Cavernous Sinus Thrombosis
}

\author{
Koichi Kobayashi and Hirotoshi Matsui
}

Key words: lemierre's syndrome, cavernous sinus thrombosis

(Intern Med 56: 887-888, 2017)

(DOI: 10.2169/internalmedicine.56.7878)

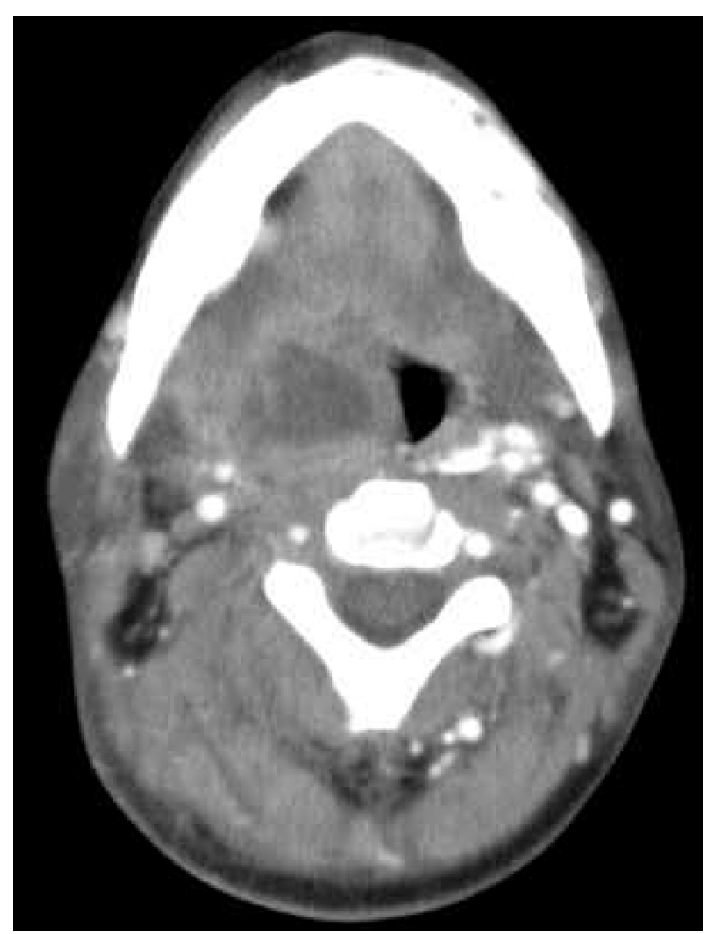

Picture 1.

A 46-year-old man was referred to our institution due to hemoptysis and a fever. He had no notable medical history except for schizophrenia. Limited mouth opening due to severe pharyngeal pain and right conjunctival edema were remarkable, as well as septic shock. Computed tomography revealed peritonsillar abscess (Picture 1) and septic pulmonary embolisms (Picture 2). Pus from the abscess and blood culture were positive for Prevotella intermedia. While antibiotics and drainage improved his general status, leading to a recovery from septic shock, the conjunctival edema worsened, and the dysfunction of the cranial nerve III, IV, VI emerged. Magnetic resonance imaging revealed thrombus formation within the right internal jugular vein (Picture 3; big arrow) extending to the sigmoid sinus (arrowhead) and
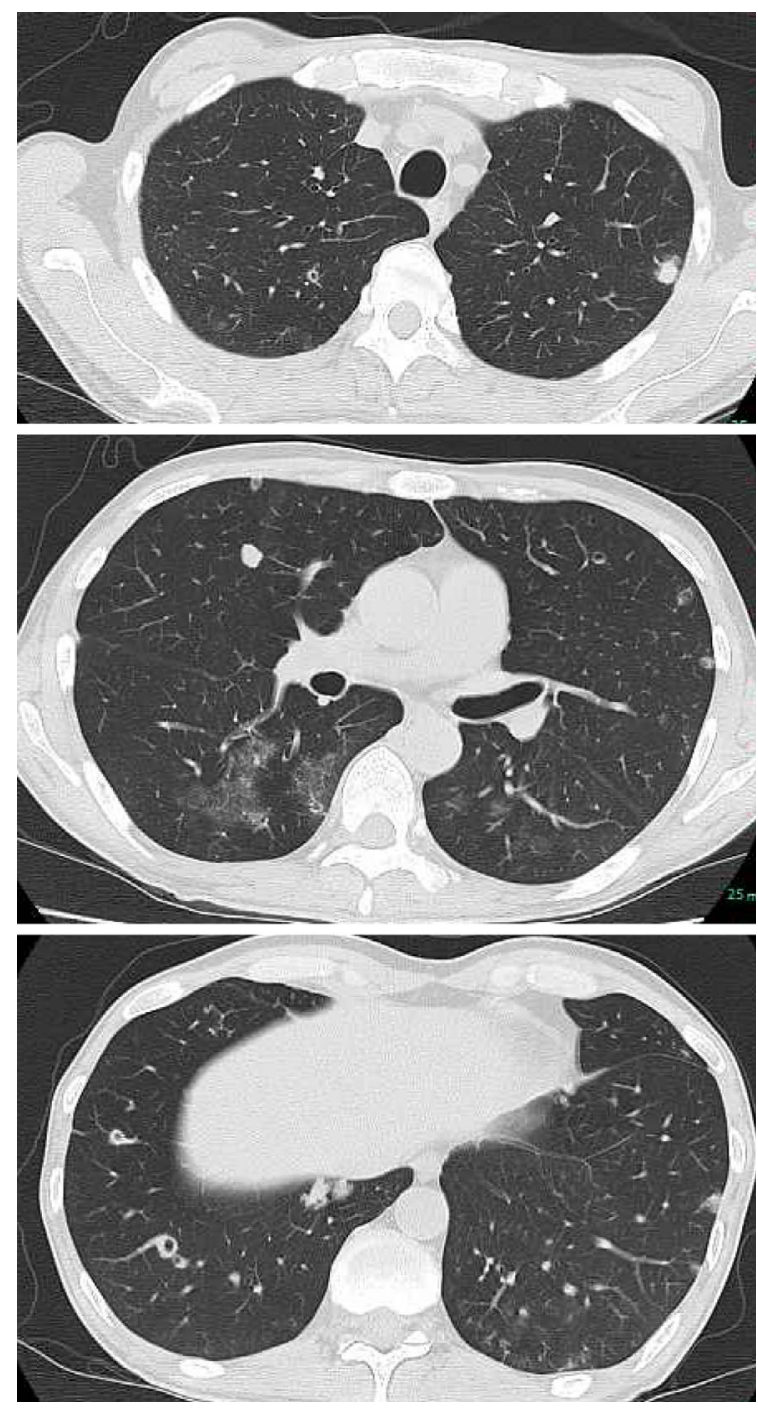

Picture 2.

even to the cavernous sinus (small arrow). He was diagnosed with Lemierre's syndrome based on the presence of disseminated abscesses and internal jugular vein thrombo- 

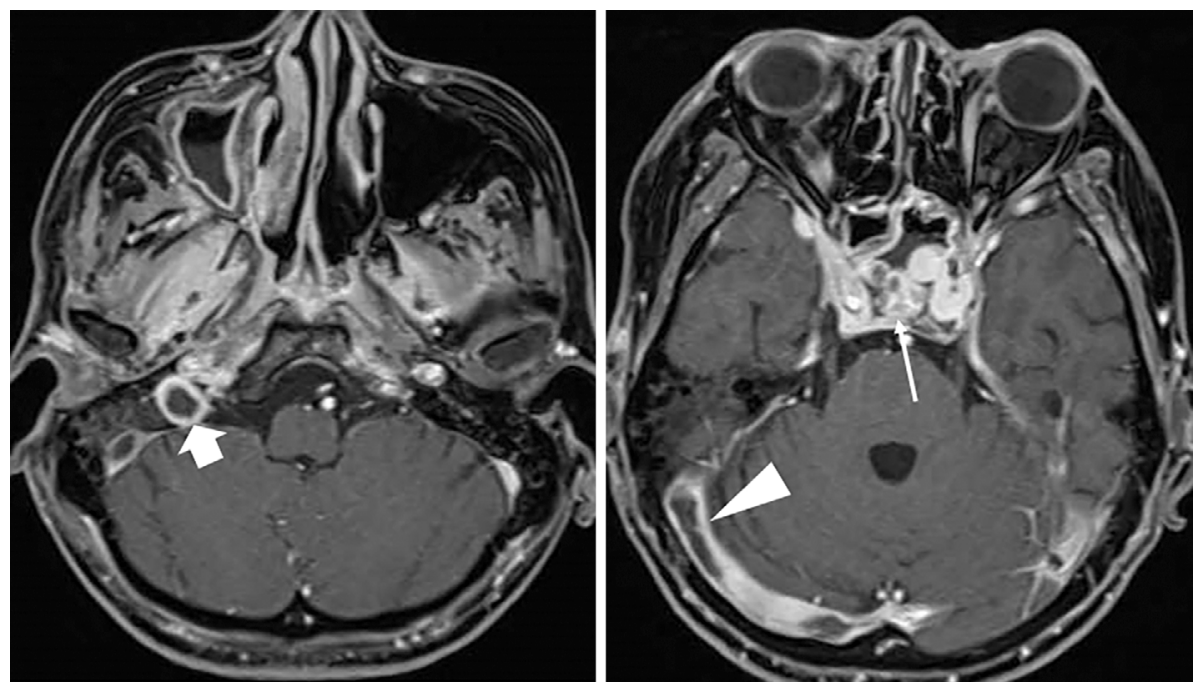

Picture 3.

phlebitis after developing an infection of the oropharynx (1). Anticoagulant therapy with heparin could not fully improve the neurological impairment.

The authors state that they have no Conflict of Interest (COI).

\section{Reference}

1. Lai C, Vummidi DR. Images in clinical medicine. Lemierre's syndrome. N Engl J Med 350: e14, 2004.

The Internal Medicine is an Open Access article distributed under the Creative Commons Attribution-NonCommercial-NoDerivatives 4.0 International License. To view the details of this license, please visit (https://creativecommons.org/licenses/ by-nc-nd/4.0/).

(C) 2017 The Japanese Society of Internal Medicine http://www.naika.or.jp/imonline/index.html 